Department of Econometrics and Business Statistics

http://monash.edu/business/ebs/research/publications

\title{
Fast forecast reconciliation using linear models
}

Mahsa Ashouri, Rob J Hyndman, Galit Shmueli

December 2019

Working Paper 29/19 


\title{
Fast forecast reconciliation using linear models
}

\author{
Mahsa Ashouri \\ Institute of Service Science, National Tsing Hua University, Taiwan \\ Email: mahsa.ashouri@iss.nthu.edu.tw \\ Corresponding author
}

\section{Rob J Hyndman}

Monash University, Clayton VIC 3800, Australia

Email: rob.hyndman@monash.edu

\section{Galit Shmueli}

Institute of Service Science, National Tsing Hua University, Taiwan Email: galit.shmueli@iss.nthu.edu.tw

3 December 2019

JEL classification: $\mathrm{C} 10, \mathrm{C} 14, \mathrm{C} 22$ 


\title{
Fast forecast reconciliation using linear models
}

\begin{abstract}
Forecasting hierarchical or grouped time series usually involves two steps: computing base forecasts and reconciling the forecasts. Base forecasts can be computed by popular time series forecasting methods such as Exponential Smoothing (ETS) and Autoregressive Integrated Moving Average (ARIMA) models. The reconciliation step is a linear process that adjusts the base forecasts to ensure they are coherent. However using ETS or ARIMA for base forecasts can be computationally challenging when there are a large number of series to forecast, as each model must be numerically optimized for each series. We propose a linear model that avoids this computational problem and handles the forecasting and reconciliation in a single step. The proposed method is very flexible in incorporating external data, handling missing values and model selection. We illustrate our approach using two datasets: monthly Australian domestic tourism and daily Wikipedia pageviews. We compare our approach to reconciliation using ETS and ARIMA, and show that our approach is much faster while providing similar levels of forecast accuracy.
\end{abstract}

Keywords: hierarchical forecasting, grouped forecasting, reconciling forecast, linear regression

\section{Introduction}

Modern data collection tools have dramatically increased the amount of available time series data. For example, the Internet of Things and point-of-sale scanning produce huge volumes of time series in a short period of time. Naturally, there is an interest in forecasting these time series, yet forecasting large collections of time series is computationally challenging.

\subsection{Hierarchical and grouped time series}

In many cases, these time series can be structured and disaggregated based on hierarchies or groups such as geographic location, product type, gender, etc. An example of hierarchical time series is sales in restaurant chains, which can be disaggregated into different stores and then different types of food or drinks. Figure 1 shows a schematic of such a hierarchical time series 
structure with three levels. The top level is the total series, formed by aggregating all the bottom level series. In the middle level, series are aggregations of their own child series; for instance, series A is the aggregation of AW and AX. Finally, the bottom level series, includes the most disaggregated series.

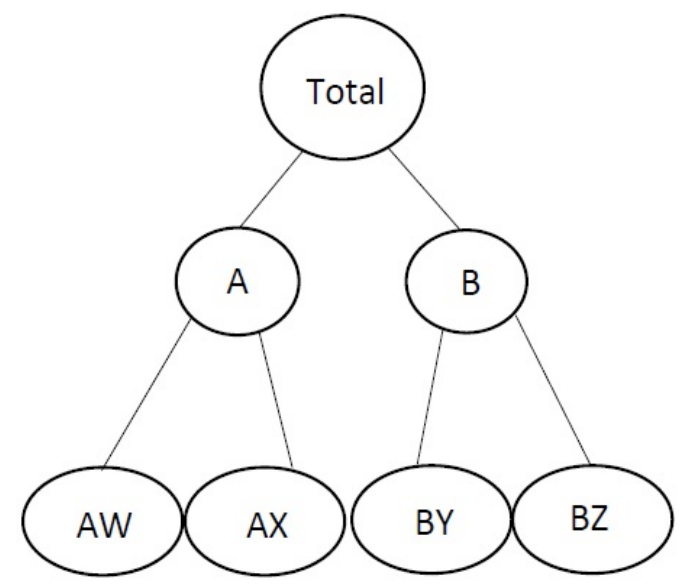

Figure 1: An example of a two level hierarchical structure.

Grouped time series involve more complicated aggregation structures compared to strictly hierarchical time series. To take the simplest example, suppose we have two grouping factors which are not nested: sex (Male/Female) and city (New York/San Francisco). The disaggregated series for each combination of sex and city can be combined to form city sub-totals, or sex sub-totals. These sub-totals can be combined to give the overall total. Both sub-totals are of interest.

We can think of such structures as hierarchical time series without a unique hierarchy. A schematic of this grouped time series structure is shown in Figure 2 with two grouping factors, each of two levels (A/B and C/D). The series in this structure can be split first into groups A and $B$ and then subdivided further into $C$ and D (left side), or split first into $C$ and $D$ and then subdivided into A and B (right side). The final disaggregation is identical in both cases, but the middle level aggregates are different.

We use the same notation (following Hyndman \& Athanasopoulos 2018) for both hierarchical and grouped time series. We denote the total series at time $t$ by $y_{t}$, and the series at node $Z$ (subaggregation level $Z$ ) and time $t$ by $y_{Z, t}$. For describing the relationships between series, we use an $N \times M$ matrix, called the "summing matrix", denoted by $S$, in which $N$ is the overall number of nodes and $M$ is the number of bottom level nodes. For example in Figure $1, N=7$ and $M=4$, while in Figure $2, N=9$ and $M=4$. Then we can write $\boldsymbol{y}_{t}=\boldsymbol{S} \boldsymbol{b}_{t}$, where $\boldsymbol{y}_{t}$ is a vector of all the level nodes at time $t$ and $\boldsymbol{b}_{t}$ is the vector of all the bottom level nodes at time $t$. 

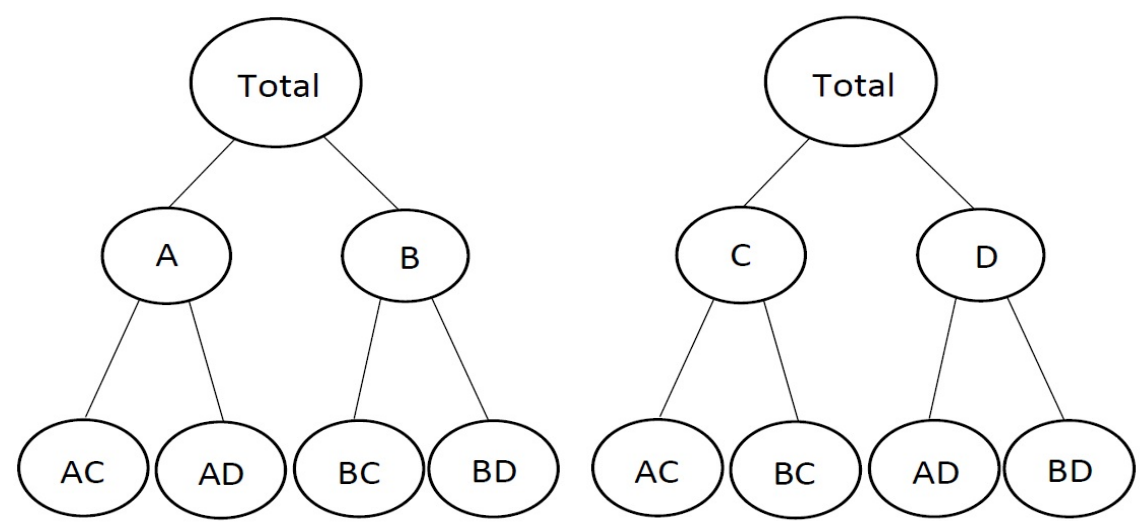

Figure 2: An example of a two level grouped structure.

For the example shown in Figure 2, the equation can be written as follows:

$$
\left(\begin{array}{c}
y_{t} \\
y_{A, t} \\
y_{B, t} \\
y_{C, t} \\
y_{D, t} \\
y_{A C, t} \\
y_{A D, t} \\
y_{B C, t} \\
y_{B D, t}
\end{array}\right)=\left(\begin{array}{llll}
1 & 1 & 1 & 1 \\
1 & 1 & 0 & 0 \\
0 & 0 & 1 & 1 \\
1 & 0 & 1 & 0 \\
0 & 1 & 0 & 1 \\
1 & 0 & 0 & 0 \\
0 & 1 & 0 & 0 \\
0 & 0 & 1 & 0 \\
0 & 0 & 0 & 1
\end{array}\right)\left(\begin{array}{l}
y_{A C, t} \\
y_{A D, t} \\
y_{B C, t} \\
y_{B D, t}
\end{array}\right) .
$$

\subsection{Forecasting hierarchical time series}

If we just forecast each series individually, we are ignoring the hierarchical or grouping structure, and the forecasts will not be "coherent" (they will not add up appropriately).

There are several available methods that consider the hierarchical structure information when forecasting time series. These include the top-down (Gross \& Sohl 1990; Fliedner 2001), bottomup (Kahn 1998), middle-out and optimal combination (Hyndman et al. 2011) approaches. In the top-down approach, we first forecast the total series and then disaggregate the forecast to form lower level series forecasts based on a set of historical and forecasted proportions (for details see Athanasopoulos, Ahmed \& Hyndman 2009). In the bottom-up approach, the forecasts in each level of the hierarchy can be computed by aggregating the bottom level series forecasts. However, we may not get good upper-level forecasts because the most disaggregated series can 
be noisy and so their forecasts are often inaccurate. In the middle-out approach, the process can be started from one of the middle levels and other forecasts can be computed using aggregation for upper levels and disaggregation for lower levels. Finally, optimal combination uses all the $n$ forecasts for all of the series in the entire structure, and then uses an optimization process to reconcile the resulting forecasts. The advantage of the optimal combination method, compared with the other methods, is that it considers all information in the hierarchy, including any correlations among the series.

In the optimal combination method, reconciled forecasts can be computed using the following equation known as weighted least squares (WLS) (Wickramasuriya, Athanasopoulos \& Hyndman 2019)

$$
\tilde{\boldsymbol{y}}_{h}=\boldsymbol{S}\left(\boldsymbol{S}^{\prime} \boldsymbol{W}_{h}^{-1} \boldsymbol{S}\right)^{-1} \boldsymbol{S}^{\prime} \boldsymbol{W}_{h}^{-1} \hat{\boldsymbol{y}}_{h}
$$

where $\hat{y}_{h}$ represents a vector of $h$-step-ahead base forecasts for all levels of the hierarchy, and $W_{h}$ is the covariance matrix of forecast errors for the $h$-step-ahead base forecasts.

Several possible simple methods for estimating $\boldsymbol{W}_{h}$ are available. Wickramasuriya, Athanasopoulos \& Hyndman (2019) discuss a simple approximation whereby $\boldsymbol{W}_{h}=k_{h} \boldsymbol{\Lambda}$ with $k_{h}$ being a positive constant, $\Lambda=\operatorname{diag}(\mathbf{S 1})$, and $\mathbf{1}$ being a column of 1 s. Note that $\Lambda$ simply contains the row sums of the summing matrix $S$, and that $k_{h}$ will cancel out in (1). Thus

$$
\tilde{\boldsymbol{y}}_{h}=\boldsymbol{S}\left(\boldsymbol{S}^{\prime} \boldsymbol{\Lambda}^{-1} \boldsymbol{S}\right)^{-1} \boldsymbol{S}^{\prime} \boldsymbol{\Lambda}^{-1} \hat{\boldsymbol{y}}_{h}
$$

The most computationally challenging part of the optimal combination method is to produce all the base forecasts that make up $\hat{y}_{h}$. In many applications, there may be thousands or even millions of individual series, and each of them must be forecast independently. The most popular time series forecasting methods such as ETS and ARIMA models (Hyndman \& Athanasopoulos 2018) involve non-linear optimization routines to estimate the parameters via maximum likelihood estimation. Usually, multiple models are fitted for each series, and the best is select by minimizing Akaike's Information Citerion (Akaike 1998). This computational challenges increases with the number of lower level series as well as in the number of aggregations of interest.

We therefore propose a new approach to compute the base forecasts that is both computationally fast while maintaining an acceptable forecasting accuracy level. 


\section{Proposed approach: Linear model}

Our proposed approach is based on using linear regression models for computing base forecasts. Suppose we have a linear model that we use for forecasting, and we wish to apply it to $N$ different series which have some aggregation constraints. We have observations $y_{t, i}$ from times $t=1, \ldots, T$ and series $i=1, \ldots, N$. Then

$$
y_{t, i}=\boldsymbol{\beta}_{i}^{\prime} x_{t, i}+\varepsilon_{t, i}
$$

where $x_{t, i}=\left\{1, x_{t, i, 1}, \ldots, x_{t, i, p}\right\}$ is a $(p+1)$-vector of regression variables. This equation for all the observations in matrix form can be written as follows:

$$
\left(\begin{array}{c}
y_{1} \\
y_{2} \\
y_{3} \\
\vdots \\
y_{N}
\end{array}\right)=\left(\begin{array}{ccccc}
X_{1} & 0 & 0 & \ldots & 0 \\
0 & X_{2} & 0 & \ldots & 0 \\
0 & 0 & X_{3} & \ddots & \vdots \\
\vdots & \vdots & \ddots & \ddots & 0 \\
0 & 0 & \ldots & 0 & X_{N}
\end{array}\right)\left(\begin{array}{c}
\beta_{1} \\
\beta_{2} \\
\beta_{3} \\
\vdots \\
\beta_{N}
\end{array}\right)+\left(\begin{array}{c}
\varepsilon_{1} \\
\varepsilon_{2} \\
\varepsilon_{3} \\
\vdots \\
\varepsilon_{N}
\end{array}\right)
$$

where $\boldsymbol{y}_{i}=\left\{y_{1, i}, y_{2, i}, \ldots, y_{T, i}\right\}$ is a $T$-vector, $\boldsymbol{\beta}_{i}=\left\{\beta_{0, i}, \beta_{1, i}, \beta_{2, i}, \ldots, \beta_{p, i}\right\}$ is a $(p+1)$-vector, $\varepsilon_{i}=\left\{\varepsilon_{1, i}, \varepsilon_{2, i}, \ldots, \varepsilon_{T, i}\right\}$ is a $T$-vector and $X_{i}$ is the $T \times(p+1)$-matrix

$$
\boldsymbol{X}_{i}=\left(\begin{array}{ccccc}
1 & x_{1, i, 1} & x_{1, i, 2} & \ldots & x_{1, i, p} \\
1 & x_{2, i, 1} & x_{2, i, 2} & \ldots & x_{2, i, p} \\
\vdots & \vdots & \vdots & & \vdots \\
1 & x_{T, i, 1} & x_{T, i, 2} & \ldots & x_{T, i, p}
\end{array}\right)
$$

Equation (3) can be written as $\boldsymbol{Y}=\boldsymbol{X} \boldsymbol{B}+\boldsymbol{E}$, with parameter estimates given by $\hat{\boldsymbol{B}}=\left(\boldsymbol{X}^{\prime} \boldsymbol{X}\right)^{-1} \boldsymbol{X}^{\prime} \boldsymbol{Y}$. Then the base forecasts are obtained using

$$
\hat{\boldsymbol{y}}_{t+h}=\boldsymbol{X}_{t+h}^{*} \hat{\boldsymbol{B}}
$$

where $\hat{\boldsymbol{y}}_{t+h}$ is an $N$-vector of forecasts, $\hat{\boldsymbol{B}}$ comprises $N$ stacked $(p+1)$-vectors of estimated coefficients, and $\boldsymbol{X}_{t+h}^{*}$ is the $N \times N(p+1)$ matrix 


$$
\boldsymbol{X}_{t+h}^{*}=\left(\begin{array}{ccccc}
x_{t+h, 1}^{\prime} & 0 & 0 & \ldots & 0 \\
0 & x_{t+h, 2}^{\prime} & 0 & \ldots & 0 \\
0 & 0 & x_{t+h, 3}^{\prime} & \ddots & \vdots \\
\vdots & \vdots & \ddots & \ddots & 0 \\
0 & 0 & \ldots & 0 & x_{t+h, N}^{\prime}
\end{array}\right)
$$

Note that we use $\boldsymbol{X}_{t}^{*}$ to distinguish this matrix, which combines $\boldsymbol{x}_{t, i}$ across all series for one time from $\boldsymbol{X}_{i}$ which combines $\boldsymbol{x}_{t, i}$ across all time for one series.

Finally, we can combine the two linear equations for computing base forecasts and reconciled forecasts (Equations (2) and (4)) to obtain the reconciled forecasts with a single equation:

$$
\tilde{y}_{t+h}=\boldsymbol{S}\left(\boldsymbol{S}^{\prime} \boldsymbol{\Lambda} \boldsymbol{S}\right)^{-1} \boldsymbol{S}^{\prime} \boldsymbol{\Lambda}\left(\boldsymbol{X}_{t+h}^{*} \hat{\boldsymbol{B}}\right)=\boldsymbol{S}\left(\boldsymbol{S}^{\prime} \boldsymbol{\Lambda} \boldsymbol{S}\right)^{-1} \boldsymbol{S}^{\prime} \boldsymbol{\Lambda} \boldsymbol{X}_{t+h}^{*}\left(\boldsymbol{X}^{\prime} \boldsymbol{X}\right)^{-1} \boldsymbol{X}^{\prime} \boldsymbol{Y}
$$

\subsection{Simplified formulation for a fixed set of predictors (X)}

If we have the same set of predictor variables, $\boldsymbol{X}$, for all the series, we can write Equations (3) to (5) more easily using multivariate regression equations, and we can obtain all the reconciled forecasts for all the series in one equation. In that case, Equation (3) can be rearranged as follows:

$$
\left(\begin{array}{ccc}
y_{11} & \ldots & y_{1 N} \\
y_{21} & \ldots & y_{2 N} \\
\vdots & & \vdots \\
y_{T 1} & \ldots & y_{T N}
\end{array}\right)=\left(\begin{array}{cccc}
1 & X_{11} & \ldots & X_{1 p} \\
1 & X_{21} & \ldots & X_{2 p} \\
\vdots & \vdots & & \vdots \\
1 & X_{T 1} & \ldots & X_{T p}
\end{array}\right)\left(\begin{array}{ccc}
\beta_{01} & \ldots & \beta_{0 N} \\
\beta_{11} & \ldots & \beta_{1 N} \\
\vdots & & \vdots \\
\beta_{p 1} & \ldots & \beta_{p N}
\end{array}\right)+\left(\begin{array}{ccc}
\varepsilon_{11} & \ldots & \varepsilon_{1 N} \\
\varepsilon_{21} & \ldots & \varepsilon_{2 N} \\
\vdots & & \vdots \\
\varepsilon_{T 1} & \ldots & \varepsilon_{T N}
\end{array}\right)
$$

where $\boldsymbol{Y}, \boldsymbol{X}, \boldsymbol{B}$ and $\boldsymbol{E}$ are now matrices of size $T \times N, T \times(p+1),(p+1) \times N$ and $T \times N$, respectively. Equations (4) to (5) can be written accordingly using Equation (6) and here $\boldsymbol{X}_{t+h, i}^{*}=$ $\boldsymbol{X}_{t+h}^{*}$, where $\boldsymbol{X}_{t+h}^{*}$ is an $h \times(p+1)$ matrix.

\subsection{OLS predictors}

As an example of the $\boldsymbol{X}_{t}$ matrix in Equation (3), we can refer to the set of predictors proposed in Ashouri, Shmueli \& Sin (2018) for modeling trend, seasonality and autocorrelation by using lagged values $\left(y_{t-1}, y_{t-2}, \ldots\right)$, trend variables and seasonal dummy variables:

$$
y_{t}=\alpha_{0}+\alpha_{1} t+\beta_{1} s_{1, t}+\cdots+\beta_{m-1} s_{m-1, t}+\gamma_{1} y_{t-1}+\cdots+\gamma_{p} y_{t-p}+\delta z_{t}+\varepsilon_{t} .
$$


Here, $s_{j, t}$ is a dummy variable taking value 1 if time $t$ is in season $j(j=1,2, \ldots, m), y_{t-k}$ is the $k$ th lagged value for $y_{t}$ and $z_{t}$ is some external information at time $t$. The seasonal period $m$ depends on the problem; for instance, if we have daily data with day-of-week seasonality, then $m=7$.

Because of using lags and external series as predictors in Equation (7), we do not have same set of predictors for all the series, $y_{t}$. However, if we just use trend and seasonality dummies as the predictors, then the simpler equations given in Section 2 can be written using multivariate regression models.

While OLS is popular in practice for forecasting time series, it is often frowned upon due to its independence assumption. This can cause issues for parametric inference but is less of a problem for forecasting. In fact it often performs sufficiently well for forecasting as can be seen by its popular use in practice. Further, the use of autoregressive terms in the above model should model most of the autocorrelation in the data.

\subsection{Computational considerations}

There are two ways for computing the above forecasts. First, we could create the matrices $Y, X$ and $E$, and then directly use the above equations (taking advantage of sparse matrix routines) to obtain the forecasts. Alternatively, we could use separate regression models to compute the coefficients for each linear model individually. Although the matrix, $\boldsymbol{X}^{\prime} \boldsymbol{X}$, which we need to invert is sparse and block diagonal, it is still faster to use the second approach involving separate regression models.

\subsection{Prediction intervals}

For obtaining prediction intervals, we need to compute the variance of reconciled forecasts as follows (Wickramasuriya, Athanasopoulos \& Hyndman 2019):

$$
\operatorname{Var}\left(\tilde{\boldsymbol{y}}_{t+h}\right)=\boldsymbol{S P} \boldsymbol{\Sigma}_{t+h} \boldsymbol{P}^{\prime} \boldsymbol{S}^{\prime}
$$

where $\boldsymbol{P}=\left(\boldsymbol{S}^{\prime} \boldsymbol{\Lambda} \boldsymbol{S}\right)^{-1} \boldsymbol{S}^{\prime} \boldsymbol{\Lambda}$ and $\boldsymbol{\Sigma}_{t+h}$ denotes the variance of the base forecasts given by the usual linear model formula (Hyndman \& Athanasopoulos 2018)

$$
\boldsymbol{\Sigma}_{t+h}=\sigma^{2}\left[1+\boldsymbol{X}_{t+h}^{*}\left(\boldsymbol{X}^{\prime} \boldsymbol{X}\right)^{-1}\left(\boldsymbol{X}_{t+h}^{*}\right)^{\prime}\right]
$$


Assuming normally distributed errors, we can easily obtain any required prediction intervals corresponding to elements of $\boldsymbol{y}_{t+h}$ using the diagonals of (8).

\section{Applications}

In this section we illustrate our approach using two examples: forecasting monthly Australian domestic tourism and forecasting daily Wikipedia pageviews. We compare the forecasting accuracy of ETS, ARIMA and the proposed linear OLS forecasting model, with and without the reconciliation step. In these applications, we used the weighted reconciliation approach from Equation (2). For comparing these methods, we use the average of Root Mean Square Errors (RMSEs) across all series and also display box plots for forecast errors along with the raw forecast errors.

The two datasets differ in terms of structure, size and behavior. The tourism data contains 304 series with both hierarchical and grouped structure, while the Wikipedia pageviews dataset contains 913 series with grouped structure. The tourism dataset has strong seasonality while the Wikipedia data are noiser.

We apply two methods for generating forecasts, which differ in how they handle unobserved lagged values as inputs. The first approach is ex post in that it uses actual values, even when they are future to the forecast origin. These values are known to us because they are in the test set. We call these rolling origin forecasts. In the second ex ante method, we replace lagged values of $y$ by their forecasts if they occur at periods after the forecast origin. We call these fixed origin forecasts.

\subsection{Australian domestic tourism}

This dataset has 19 years of monthly visitor nights in Australia by Australian tourists, a measure used as an indicator of tourism activity (Wickramasuriya, Athanasopoulos \& Hyndman 2019). The data were collected by computer-assisted telephone interviews with 120,000 Australians aged 15 and over (Tourism Research Australia 2005). The dataset includes 304 time series each of length 228 observations. The hierarchy and grouping structure for this dataset is made using geographic and purpose of travel information.

Table 1: Australia geographic hierarchical structure.

\begin{tabular}{|c|c|c|c|c|c|}
\hline Series & Name & Label & Series & Name & Label \\
\hline
\end{tabular}




\begin{tabular}{|c|c|c|c|c|c|}
\hline Total & & & ion & & \\
\hline 1 & Australia & Total & 55 & Lakes & BCA \\
\hline State & & & 56 & Gippsland & BCB \\
\hline 2 & NSW & A & 57 & Phillip Island & $\mathrm{BCC}$ \\
\hline 3 & VIC & B & 58 & General Murray & BDA \\
\hline 4 & QLD & $\mathrm{C}$ & 59 & Goulburn & BDB \\
\hline 5 & SA & $\mathrm{D}$ & 60 & High Country & BDC \\
\hline 6 & WA & $\mathrm{E}$ & 61 & Melbourne East & BDD \\
\hline 7 & TAS & $\mathrm{F}$ & 62 & Upper Yarra & BDE \\
\hline 8 & NT & G & 63 & Murray East & BDF \\
\hline Zone & & & 64 & Wimmera+Mallee & BEA \\
\hline 9 & Metro NSW & $\mathrm{AA}$ & 65 & Western Grampians & BEB \\
\hline 10 & Nth Coast NSW & $\mathrm{AB}$ & 66 & Bendigo Loddon & BEC \\
\hline 11 & Sth Coast NSW & $\mathrm{AC}$ & 67 & Macedon & BED \\
\hline 12 & Sth NSW & $\mathrm{AD}$ & 68 & Spa Country & BEE \\
\hline 13 & Nth NSW & $\mathrm{AE}$ & 69 & Ballarat & BEF \\
\hline 14 & $\mathrm{ACT}$ & $\mathrm{AF}$ & 70 & Central Highlands & BEG \\
\hline 15 & Metro VIC & BA & 71 & Gold Coast & CAA \\
\hline 16 & West Coast VIC & $\mathrm{BB}$ & 72 & Brisbane & $\mathrm{CAB}$ \\
\hline 17 & East Coast VIC & $\mathrm{BC}$ & 73 & Sunshine Coast & CAC \\
\hline 18 & Nth East VIC & $\mathrm{BD}$ & 74 & Central Queensland & CBA \\
\hline 19 & Nth West VIC & $\mathrm{BE}$ & 75 & Bundaberg & $\mathrm{CBB}$ \\
\hline 20 & Metro QLD & CA & 76 & Fraser Coast & СBC \\
\hline 21 & Central Coast QLD & $\mathrm{CB}$ & 77 & Mackay & CBD \\
\hline 22 & Nth Coast QLD & $\mathrm{CC}$ & 78 & Whitsundays & CCA \\
\hline 23 & Inland QLD & $\mathrm{CD}$ & 79 & Northern & ССВ \\
\hline 24 & Metro SA & DA & 80 & Tropical North Queensland & $\mathrm{CCC}$ \\
\hline 25 & Sth Coast SA & DB & 81 & Darling Downs & CDA \\
\hline 26 & Inland SA & $\mathrm{DC}$ & 82 & Outback & $\mathrm{CDB}$ \\
\hline 27 & West Coast SA & DD & 83 & Adelaide & DAA \\
\hline 28 & West Coast WA & EA & 84 & Barossa & $\mathrm{DAB}$ \\
\hline 29 & Nth WA & EB & 85 & Adelaide Hills & DAC \\
\hline 30 & Sth WA & $\mathrm{EC}$ & 86 & Limestone Coast & DBA \\
\hline 31 & Sth TAS & FA & 87 & Fleurieu Peninsula & DBB \\
\hline 32 & Nth East TAS & FB & 88 & Kangaroo Island & DBC \\
\hline 33 & Nth West TAS & $\mathrm{FC}$ & 89 & Murraylands & DCA \\
\hline 34 & Nth Coast NT & GA & 90 & Riverland & DCB \\
\hline 35 & Central NT & GB & 91 & Clare Valley & DCC \\
\hline Region & & & 92 & Flinders Range and Outback & DCD \\
\hline 36 & Sydney & AAA & 93 & Eyre Peninsula & DDA \\
\hline 37 & Central Coast & $\mathrm{AAB}$ & 94 & Yorke Peninsula & DDB \\
\hline 38 & Hunter & $\mathrm{ABA}$ & 95 & Australia's Coral Coast & EAA \\
\hline
\end{tabular}




\begin{tabular}{|c|c|c|c|c|c|}
\hline 39 & North Coast NSW & $\mathrm{ABB}$ & 96 & Experience Perth & $\mathrm{EAB}$ \\
\hline 40 & Northern Rivers Tropical NSW & $\mathrm{ABC}$ & 97 & Australia's SouthWest & EAC \\
\hline 41 & South Coast & $\mathrm{ACA}$ & 98 & Australia's North West & EBA \\
\hline 42 & Snowy Mountains & ADA & 99 & Australia's Golden Outback & ECA \\
\hline 43 & Capital Country & $\mathrm{ADB}$ & 100 & Hobart and the South & FAA \\
\hline 44 & The Murray & $\mathrm{ADC}$ & 101 & East Coast & FBA \\
\hline 45 & Riverina & ADD & 102 & Launceston, Tamar and the North & FBB \\
\hline 46 & Central NSW & AEA & 103 & North West & FCA \\
\hline 47 & New England North West & AEB & 104 & Wilderness West & FCB \\
\hline 48 & Outback NSW & $\mathrm{AEC}$ & 105 & Darwin & GAA \\
\hline 49 & Blue Mountains & AED & 106 & Kakadu Arnhem & GAB \\
\hline 50 & Canberra & AFA & 107 & Katherine Daly & GAC \\
\hline 51 & Melbourne & BAA & 108 & Barkly & GBA \\
\hline 52 & Peninsula & $\mathrm{BAB}$ & 109 & Lasseter & GBB \\
\hline 53 & Geelong & BAC & 110 & Alice Springs & GBC \\
\hline 54 & Western & BBA & 111 & MacDonnell & GBD \\
\hline
\end{tabular}

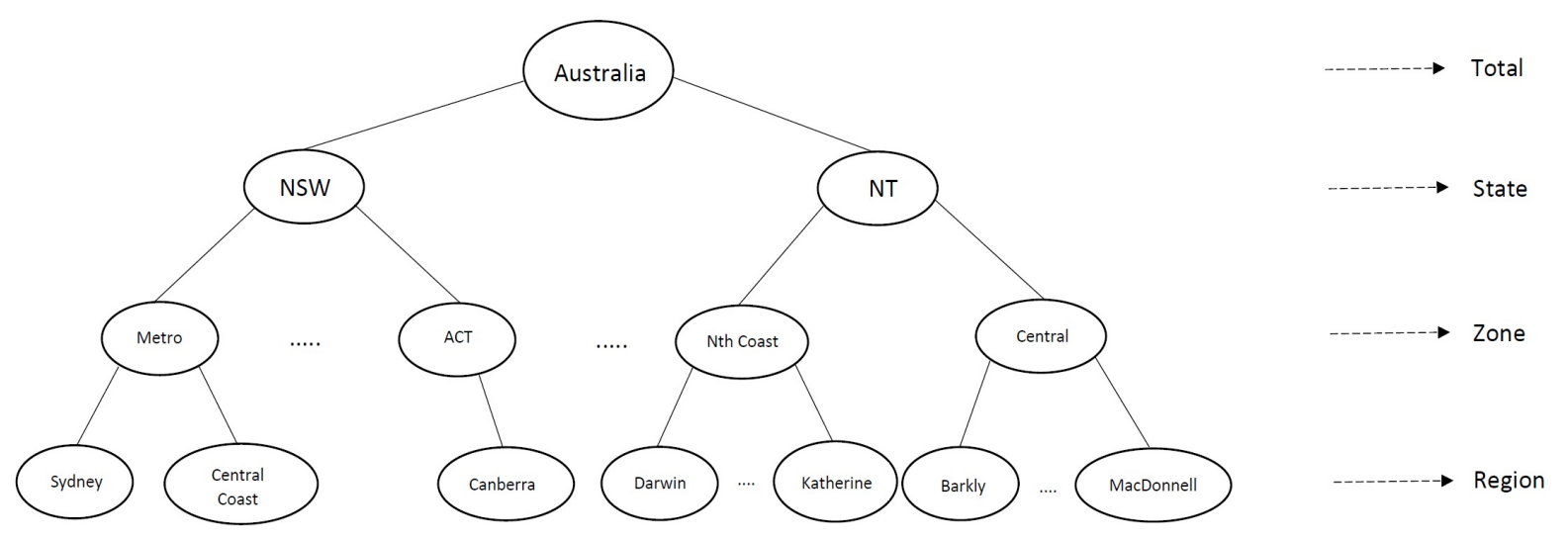

Figure 3: Australian geographic hierarchical structure.

In this dataset we have three levels of geographic divisions in Australia. In the first level, Australia is divided into seven "States" including New South Wales (NSW), Victoria (VIC), Queensland (QLD), South Australia (SA), Western Australia (WA), Tasmania (TAS) and Northern Territory (NT). In the second and third levels, it is divided into 27 "Zones" and 76 "Regions" (for details about Australia geographic divisions see Figure 3 and Table 1 and also Figure 4 which shows Australia map divided by tourism region and colored by states ${ }^{1}$ ).

We have four purposes of travel: Holiday (Hol), Visiting friends and relatives (Vis), Business (Bus) and Other (Oth). So there are $76 \times 4=304$ series at the most disaggregate level. Based on the geographic hierarchy and purpose grouping, we end up with 8 aggregation levels with 555 series in total as shown in Table 2.

\footnotetext{
${ }_{1}^{1}$ www.tra.gov.au/tra/2016/Tourism_Region_Profiles/Region_profiles/index.html
} 


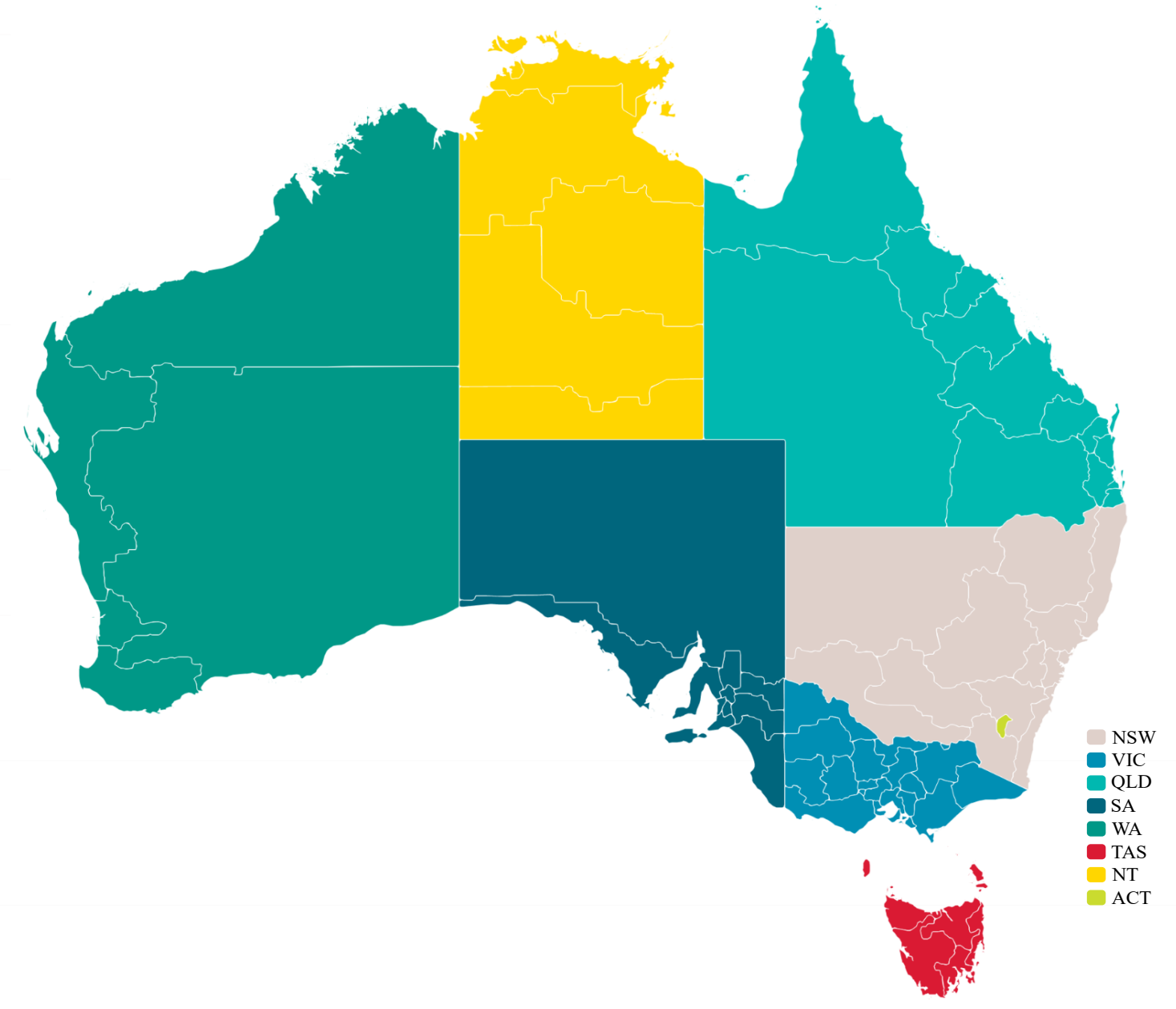

Figure 4: Australia tourism region map - colors represent states.

Table 2: Number of Australian domestic tourism series at each aggregation level.

\begin{tabular}{lr}
\hline Division & Series \\
\hline Australia & 1 \\
State & 7 \\
Zone & 27 \\
Region & 76 \\
Purpose & 4 \\
State x Purpose & 28 \\
Zone x Purpose & 108 \\
Region x Purpose & 304 \\
\hline Total & 555 \\
\hline
\end{tabular}


We report the forecast results for all these aggregation levels, as well as the average RMSE across all the levels of the hierarchy. We used different predictors in the OLS predictor matrix for the rolling and fixed origin approaches. For the rolling origin model, we included a linear trend, 11 dummy variables, and 12 lags. For the fixed origin model, we included a quadratic trend, 11 dummy variables, and lags 1 and 12. This is intended to capture the monthly seasonality. In addition, before running the model, we partition the data into training and test sets, with the last 24 months (2 years) as our test set, and the rest as our training set.

Table 3: Mean(RMSE) on 2 year test set for ETS, ARIMA and OLS with and without reconciliation Rolling origin - Tourism dataset

\begin{tabular}{lrrrrrrr}
\hline & \multicolumn{3}{c}{ Unreconciled } & & \multicolumn{3}{c}{ Reconciled } \\
\cline { 2 - 5 } \cline { 7 - 8 } Level & ETS & ARIMA & OLS & & ETS & ARIMA & OLS \\
\hline Total & 1516.4 & 1445.5 & 1415.1 & & 1517.2 & 1517.2 & 1414.7 \\
State & 511.4 & 493.1 & 510.8 & & 499.9 & 499.9 & 491.6 \\
Zone & 214.8 & 219.0 & 224.5 & & 209.6 & 209.6 & 213.6 \\
Region & 122.9 & 125.1 & 124.0 & & 119.4 & 119.4 & 120.4 \\
Purpose & 676.0 & 709.2 & 694.5 & & 674.2 & 674.2 & 681.9 \\
State x Purpose & 213.1 & 220.1 & 216.1 & & 212.7 & 212.7 & 213.3 \\
Zone x Purpose & 97.5 & 102.4 & 101.0 & & 96.8 & 96.8 & 99.0 \\
Region x Purpose & 56.2 & 58.2 & 58.2 & & 56.2 & 56.2 & 57.4 \\
\hline
\end{tabular}

Table 4: Mean(RMSE) on 2 year test set for ETS, ARIMA and OLS with and without reconciliation Fixed origin - Tourism dataset

\begin{tabular}{lrrrrrrrr}
\hline & \multicolumn{3}{c}{ Unreconciled } & & \multicolumn{3}{c}{ Reconciled } \\
\cline { 2 - 3 } \cline { 7 - 8 } Level & ETS & ARIMA & OLS & & ETS & ARIMA & OLS \\
\hline Total & 2238.6 & 3554.0 & 2528.9 & & 2232.8 & 3460.3 & 2540.1 \\
State & 593.6 & 570.1 & 596.5 & & 555.7 & 658.5 & 579.0 \\
Zone & 239.5 & 229.6 & 243.3 & & 235.3 & 249.8 & 235.4 \\
Region & 132.6 & 129.4 & 127.1 & & 127.6 & 132.4 & 123.8 \\
Purpose & 766.8 & 824.0 & 875.5 & & 801.7 & 1019.3 & 857.2 \\
State x Purpose & 226.7 & 241.2 & 236.7 & & 224.5 & 245.6 & 229.1 \\
Zone x Purpose & 103.0 & 105.4 & 104.9 & & 102.4 & 105.8 & 102.9 \\
Region x Purpose & 59.1 & 58.8 & 58.6 & & 58.8 & 59.3 & 57.9 \\
\hline
\end{tabular}

In Figures 5 and 6 we display the error box plots for both reconciled and unreconciled forecasts using all three methods, for the rolling origin and fixed origin forecasts. In these figures we see the error distributions across all the models.

Together with Tables 3 and 4, results show that our proposed OLS forecasting model produces forecast accuracy similar to ETS and ARIMA, which are computationally heavy for many time series. We also see the usefulness of the reconciliation in decreasing the average RMSE in all three methods. Except for the total series, reconciliation improves forecasts in all the hierarchy levels. 

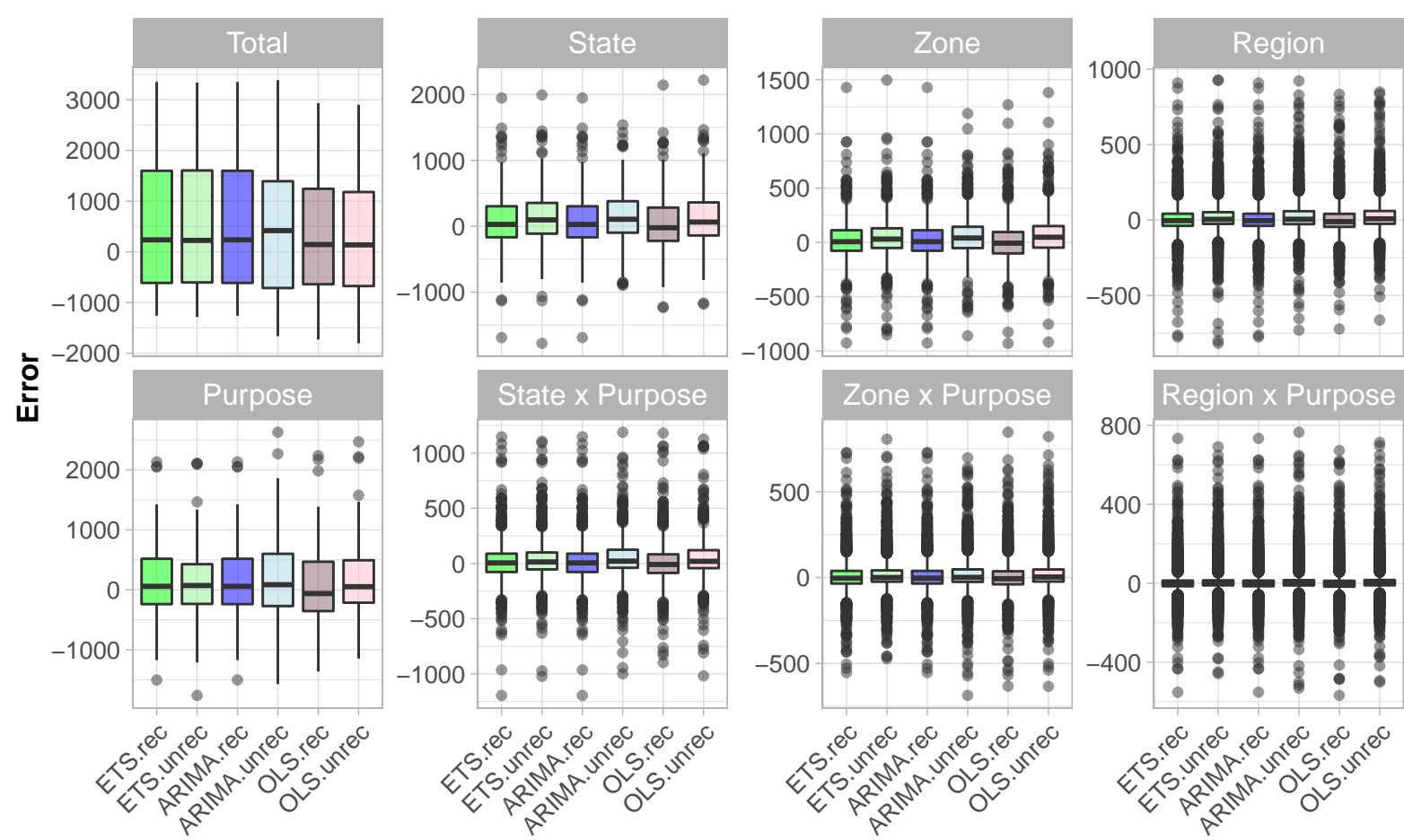

\section{Method}

Figure 5: Box plots of rolling origin forecast errors from reconciled and unreconciled ETS, ARIMA and
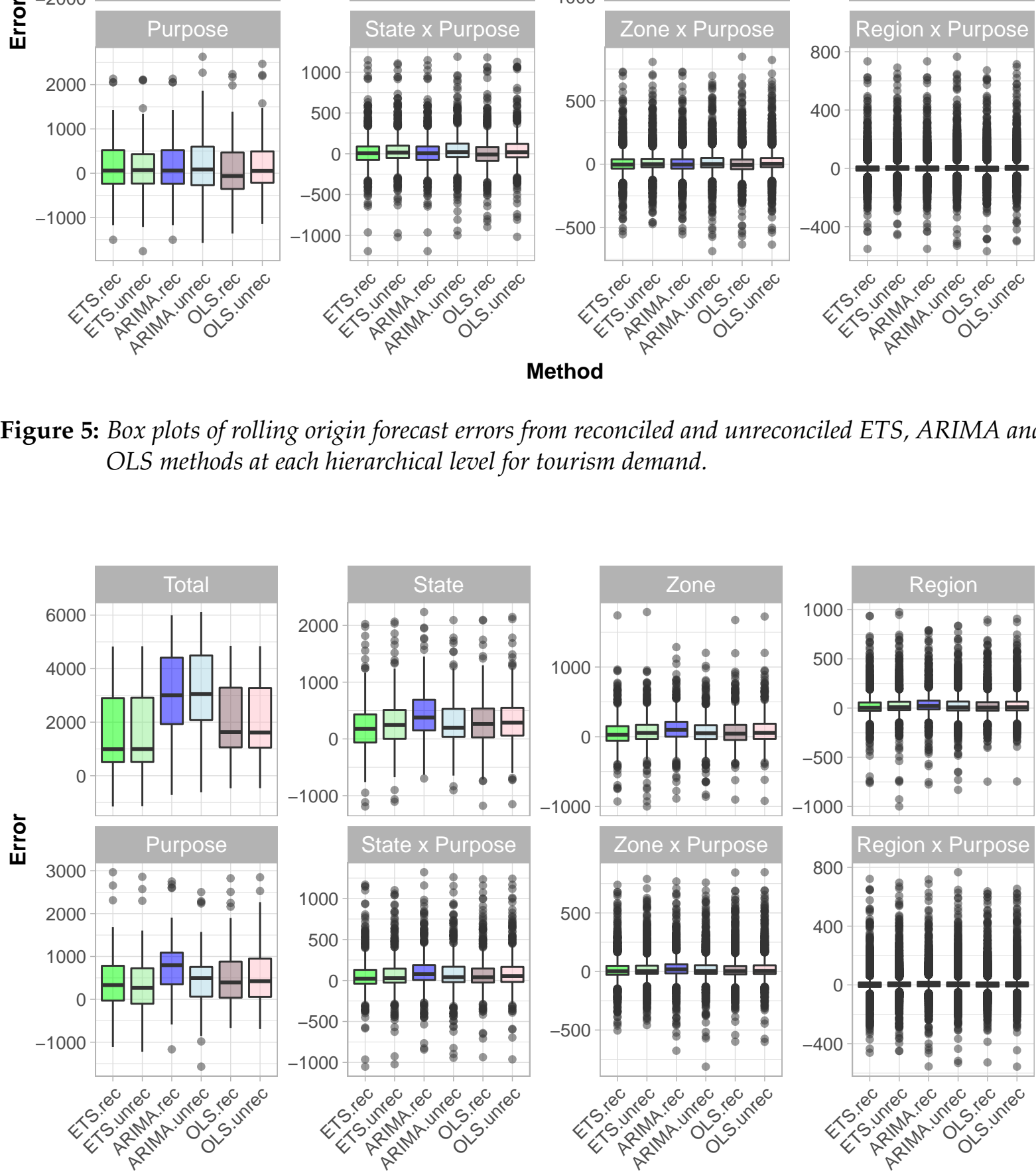

\section{OLS methods at each hierarchical level for tourism demand.}

Method

Figure 6: Box plots of fixed origin forecast errors for reconciled and unreconciled ETS, ARIMA and OLS methods at each hierarchical level for tourism demand. 
Also, because the higher level series have higher counts, the errors are larger in magnitude (Appendix A shows the box plots with scaled errors, to better compare errors across all the hierarchy levels). In addition, we see that (as expected) by applying rolling origin 1-step-ahead forecasts, the error densities are closer and more tightly distributed around zero than the fixed origin multi-step-ahead forecasts.

Figures 7 and 8 show the rolling and fixed origin forecast results for the total series and one of the bottom level series, BACBus (Geelong - Business). In these plots we have both reconciled (solid lines) and unreconciled (dashed lines) forecasts and we see that the reconciliation step improves the forecasts in this series. We also see that the OLS model forecast accuracy is similar to the other two methods.
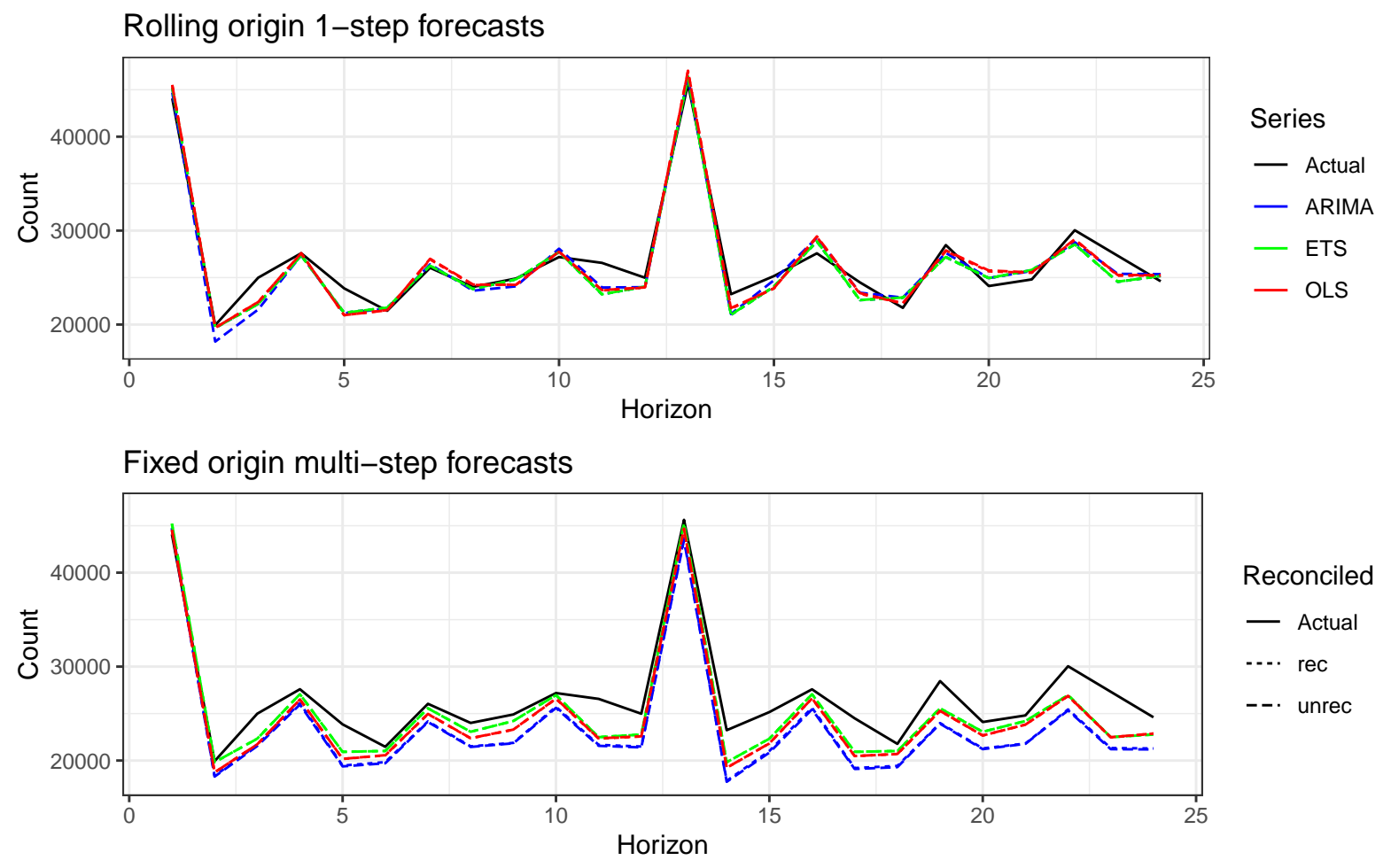

Figure 7: The actual test set for the 'Total series' compared to the forecasts from reconciled and unreconciled ETS, ARIMA and OLS methods for rolling and fixed origin tourism demand.

Table 5 compares the computation time of the three methods for rolling and fixed origin forecasting. We see that the OLS forecasting model is much faster compared to the other methods. Also, since reconciliation is a linear process, in all methods it is very fast and does not affect computation time significantly.

Since we are using a linear model, we can easily include exogenous variables which can often be helpful in improving forecast accuracy. In this application, we tried including an "Easter" 
Rolling origin 1-step forecasts

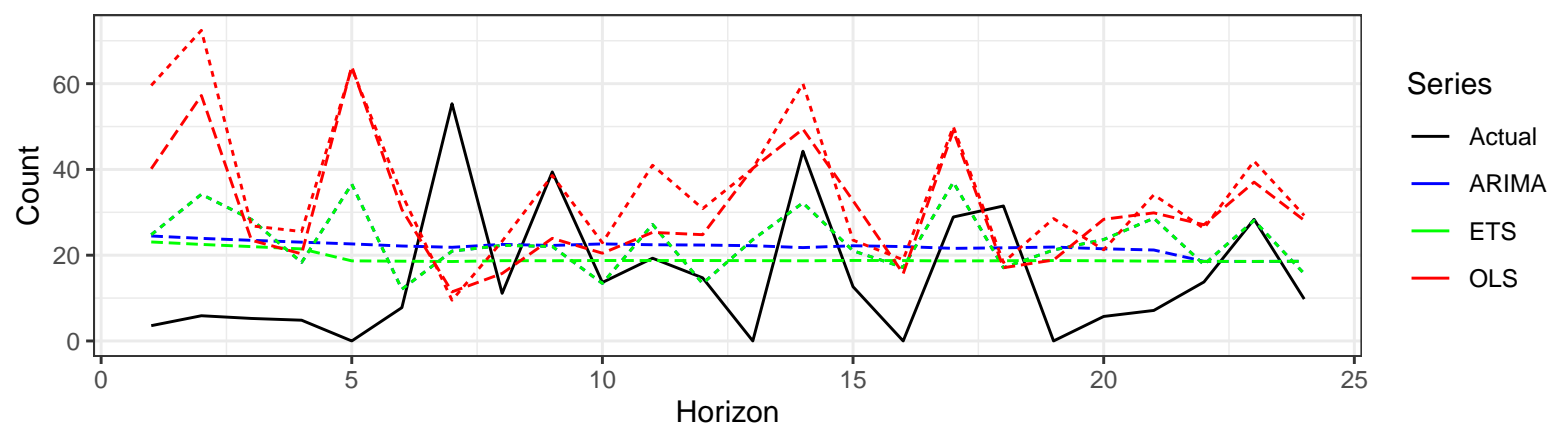

Fixed origin multi-step forecasts

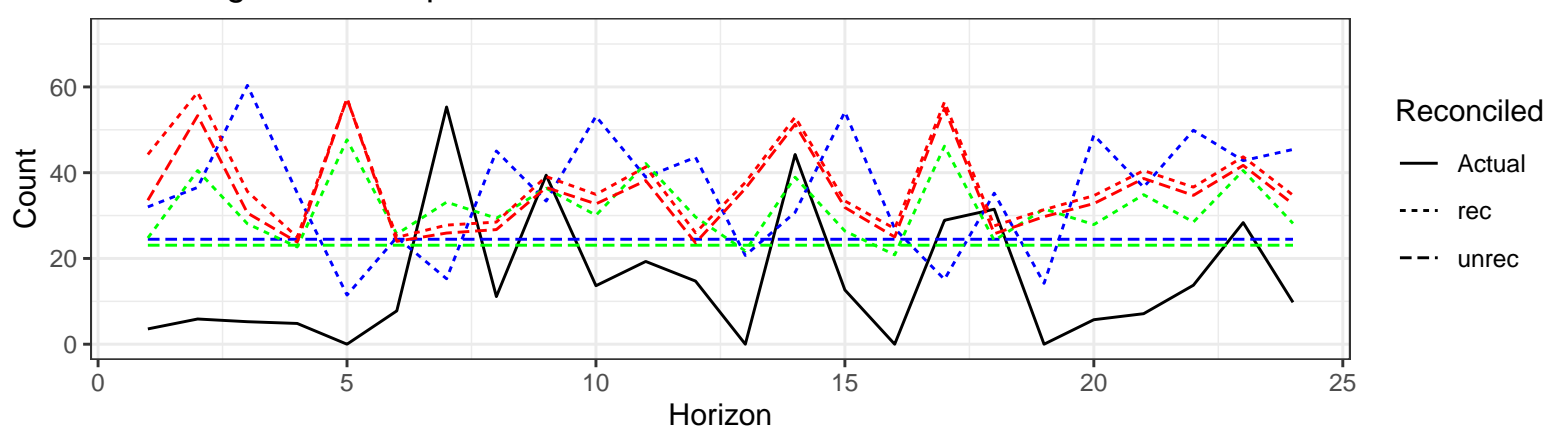

Figure 8: The actual test set for the 'BACBus' bottom level series compared to the forecasts from reconciled and unreconciled ETS, ARIMA and OLS methods for rolling and fixed origin tourism demand.

Table 5: Computation time (seconds) for ETS, ARIMA and OLS with and without reconciliation Rolling and fixed origin forecasts on a 24 month test set - Tourism dataset

\begin{tabular}{lrrrrr}
\hline & \multicolumn{2}{c}{ Rolling origin } & & \multicolumn{2}{c}{ Fixed origin } \\
\cline { 2 - 3 } \cline { 5 - 6 } & Unreconciled & Reconciled & & Unreconciled & Reconciled \\
\hline ETS & 10924.57 & 10924.60 & & 407.10 & 407.15 \\
ARIMA & 31146.38 & 31146.52 & & 1116.15 & 1116.19 \\
OLS & 48.40 & 48.31 & & 17.42 & 17.80 \\
\hline
\end{tabular}

dummy variable indicating the timing of Easter, but its affect on forecast accuracy was minimal, so it was omitted in the model reported here.

Finally, Table 6 shows that, as mentioned in Section 2.3, computation is faster using separate regression models compared to the matrix approach (even using sparse matrix algebra).

Table 6: Computation time (seconds) for OLS using the matrix approach and separate regression models, with and without reconciliation, on a rolling and fixed origin for 24 steps ahead.

\begin{tabular}{lrrrrrr}
\hline & \multicolumn{2}{c}{ Rolling origin } & & \multicolumn{2}{c}{ Fixed origin } \\
\cline { 2 - 3 } \cline { 5 - 6 } & Unreconciled & Reconciled & & Unreconciled & Reconciled \\
\hline Matrix approach & 202.06 & 209.84 & & 87.73 & 105.69 \\
Separate models & 48.40 & 48.31 & & 16.66 & 16.85 \\
\hline
\end{tabular}




\subsection{Wikipedia pageviews: Grouped structure}

The second dataset comprises one year of daily data (2016-06-01 to 2017-06-29) on Wikipedia pageviews for the most popular social networks articles (Ashouri, Shmueli \& Sin 2018). This dataset is noisier than the Australian monthly tourism data, making forecasting more challenging. The data has a grouped structure with the following attributes: "Agent": Spider, User, “Access": Desktop, Mobile app, Mobile web, “Language”: en (English), de (German), es (Spanish), zh (Chinese) and "Purpose": Blogging related, Business, Gaming, General purpose, Life style, Photo sharing, Reunion, Travel, Video (see Table 7).

Table 7: Social networking Wikipedia article grouping structure

\begin{tabular}{llll}
\hline Grouping & Series & Grouping & Series \\
\hline Total & & Language & \\
& 1. Social Network & & 10. zh (Chinese) \\
Access & & Purpose & \\
& 2. Desktop & & 11. Blogging related \\
& 3. Mobile app & 12. Business \\
Agent & & 13. Gaming \\
& 4. Mobile web & 14. General purpose \\
& 5. Spider & 15. Life style \\
Language & 6. User & 16. Photo sharing \\
& & 17. Reunion \\
& 7. en (English) & 18. Travel \\
& 8. de (German) & 19. Video \\
\hline
\end{tabular}

We consider the main aggregation factors and two-way combinations of them. The final dataset includes 913 time series, each with length 394. The applied group structure and different levels include the following aggregations: ${ }^{2}$

- Total

- Access

- Agent

- Language

- Purpose

- Access $\times$ Agent

- Access $\times$ Language

- Access $\times$ Purpose

\footnotetext{
${ }^{2}$ There are four more 3-way aggregation combinations that we do not include: Agent $\times$ Access $\times$ Language Agent $\times$ Access $\times$ Purpose, Agent $\times$ Language $\times$ Purpose, and Access $\times$ Language $\times$ Purpose. Including these four additional aggregations might slightly improve the results but for simplicity, we excluded them.
} 
- Agent $\times$ Language

- Agent $\times$ Purpose

- Language $\times$ Purpose

- Bottom level: Access $\times$ Agent $\times$ Language $\times$ Purpose

For this daily dataset, in the OLS forecasting model we include in the predictor matrix a quadratic trend, 6 seasonal dummies and all 7 lags for rolling, and for fixed origin model we use a quadratic trend, 6 seasonal dummies and lags 1 and 7 . We partitioned the data into two parts training and test sets. We used the last 28 days for our test set and the rest for the training set. In this example, the results in tables and figures are represented for single groups although we applied all the above levels in the group structure for reconciliation.

Tables 8 and 9 show the RMSE results. Although these time series are noisier, we still get acceptable results for the OLS forecasting model compared with ETS and ARIMA. In this case, we get similar results with and without the reconciliation step.

Table 8: Mean(RMSE) for ETS, ARIMA and OLS with and without reconciliation - Rolling origin Wikipedia dataset

\begin{tabular}{lrrrrrrrr}
\hline & \multicolumn{3}{c}{ Unreconciled } & & \multicolumn{3}{c}{ Reconciled } \\
\cline { 2 - 5 } \cline { 6 - 8 } Level & ETS & ARIMA & OLS & & ETS & ARIMA & OLS \\
\hline Total & 10773.7 & 15060.7 & 12288.0 & & 10851.0 & 14575.5 & 12104.3 \\
Access & 6524.7 & 6705.0 & 5915.5 & & 6314.2 & 7316.6 & 6141.8 \\
Agent & 8272.9 & 10196.3 & 8564.0 & & 7728.9 & 9896.3 & 8509.6 \\
Language & 4870.1 & 6333.0 & 5612.5 & & 5134.3 & 6372.0 & 5622.5 \\
Purpose & 5233.5 & 4659.5 & 3935.9 & & 4977.8 & 4525.3 & 3855.7 \\
Bottom level & 358.1 & 239.0 & 257.4 & & 372.3 & 245.4 & 260.1 \\
\hline
\end{tabular}

Table 9: Mean(RMSE) for ETS, ARIMA and OLS with and without reconciliation - Fixed origin Wikipedia dataset

\begin{tabular}{lrrrrrrr}
\hline & \multicolumn{3}{c}{ Unreconciled } & & \multicolumn{3}{c}{ Reconciled } \\
\cline { 2 - 3 } \cline { 6 - 8 } Level & ETS & ARIMA & OLS & & ETS & ARIMA & OLS \\
\hline Total & 14846.9 & 24298.8 & 20203.7 & & 15251.3 & 24383.6 & 20088.2 \\
Access & 7117.4 & 10732.0 & 8866.4 & & 7758.2 & 11013.9 & 8970.1 \\
Agent & 13608.7 & 17277.0 & 14985.7 & & 12431.2 & 16564.9 & 14884.8 \\
Language & 6475.9 & 9580.4 & 7913.7 & & 6728.7 & 9797.3 & 8094.7 \\
Purpose & 5302.7 & 8611.3 & 5694.1 & & 5256.3 & 8121.8 & 5665.0 \\
Bottom level & 435.6 & 389.4 & 363.7 & & 445.9 & 394.0 & 363.5 \\
\hline
\end{tabular}

Figures 9 and 10 display the forecast error box plot. These plots are for rolling and fixed origin forecasts over 28 days in each level of grouping. Further, we can see that the error distribution is almost similar in all levels across the different methods. The only exception is the Total 

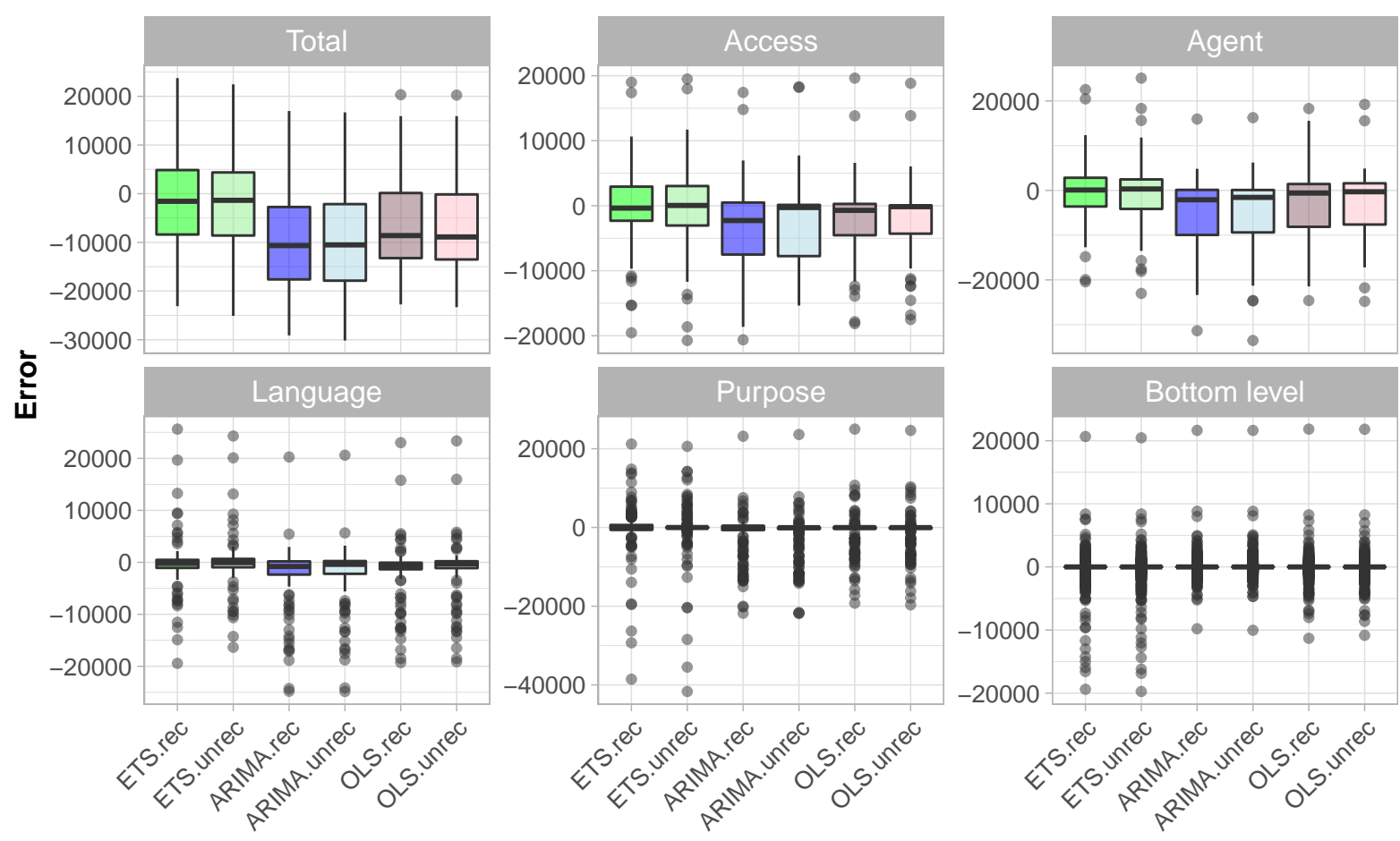

\section{Method}

Figure 9: Box plots of forecast errors for reconciled and unreconciled ETS, ARIMA and OLS methods at each hierarchical level for rolling origin forecasts of Wikipedia pageviews.
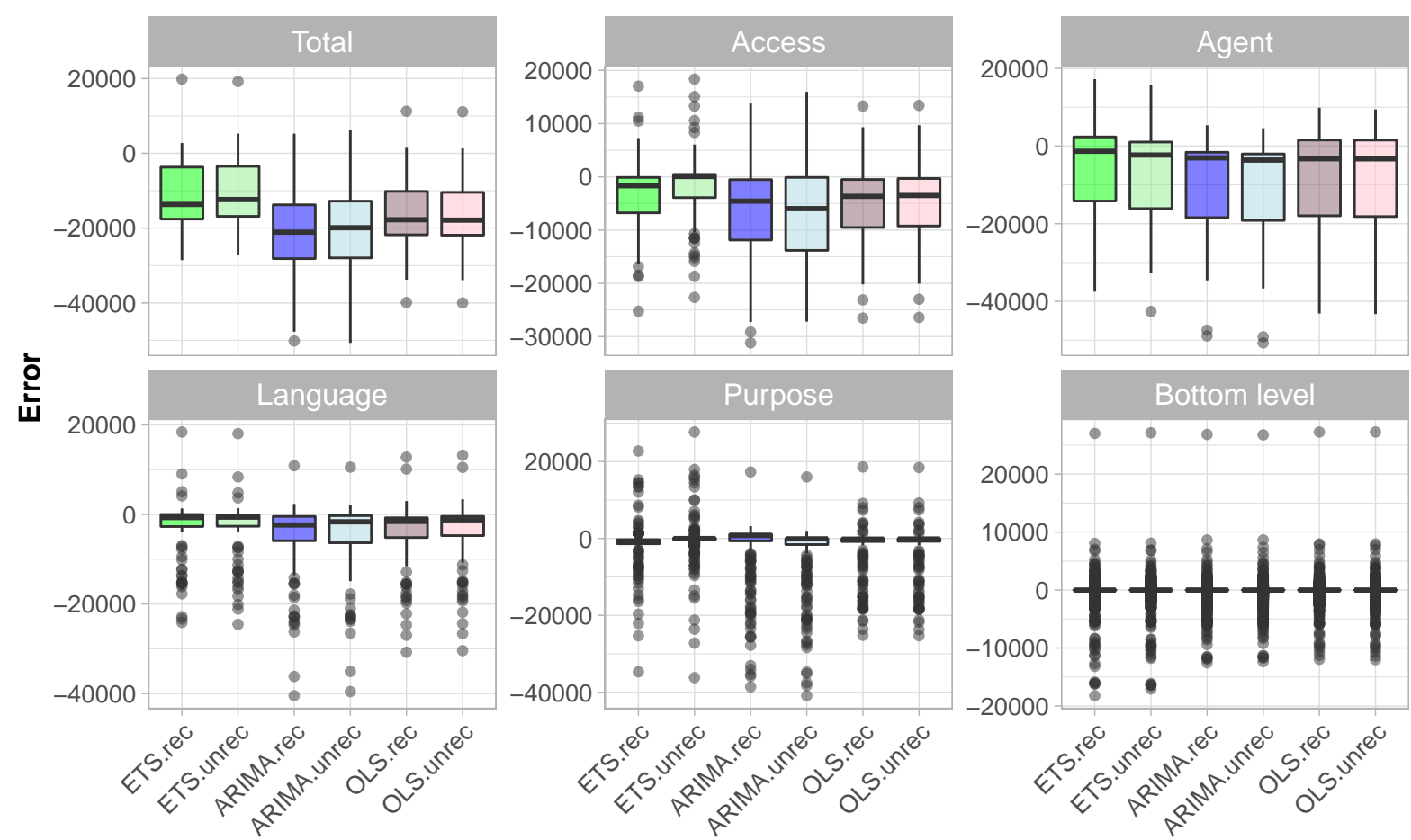

\section{Method}

Figure 10: Box plots of forecast errors for reconciled and unreconciled ETS, ARIMA and OLS methods at each hierarchical level for fixed origin forecasts of Wikipedia pageviews. 
Rolling origin 1-step forecasts

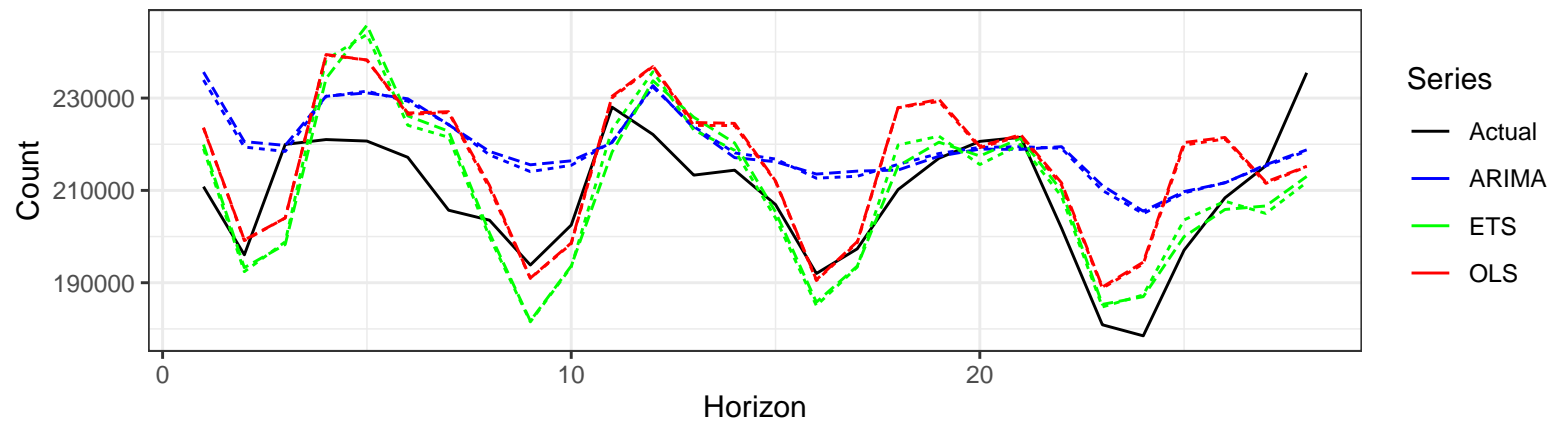

Fixed origin multi-step forecasts

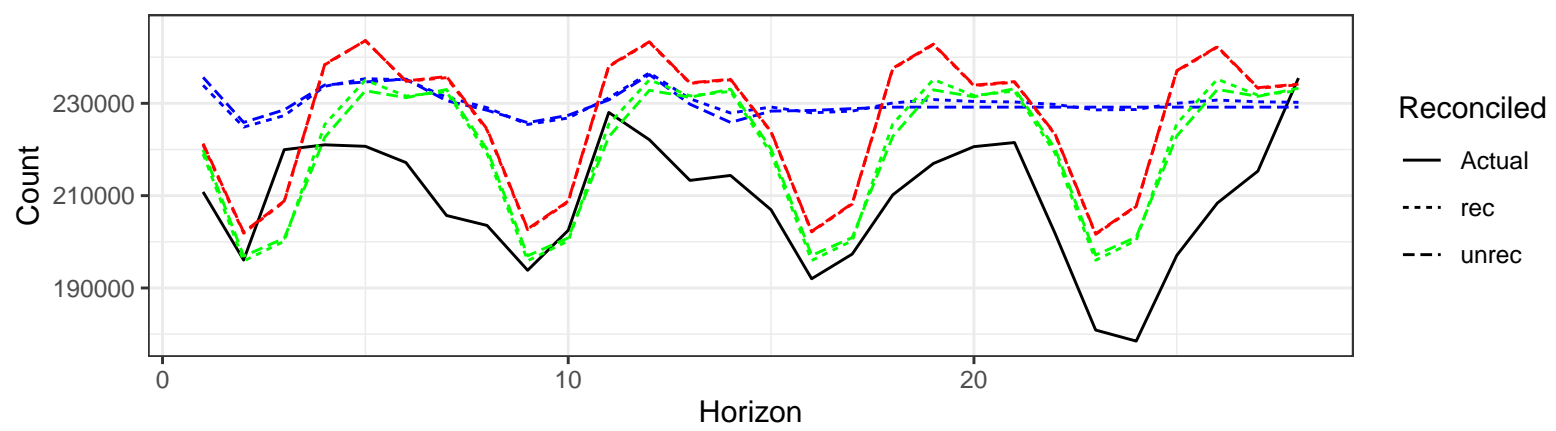

Figure 11: The actual test set for the 'Total' series compared to the forecasts from reconciled and unreconciled ETS, ARIMA and OLS methods for rolling and fixed origin forecasts of Wikipedia pageviews.

Rolling origin 1-step forecasts

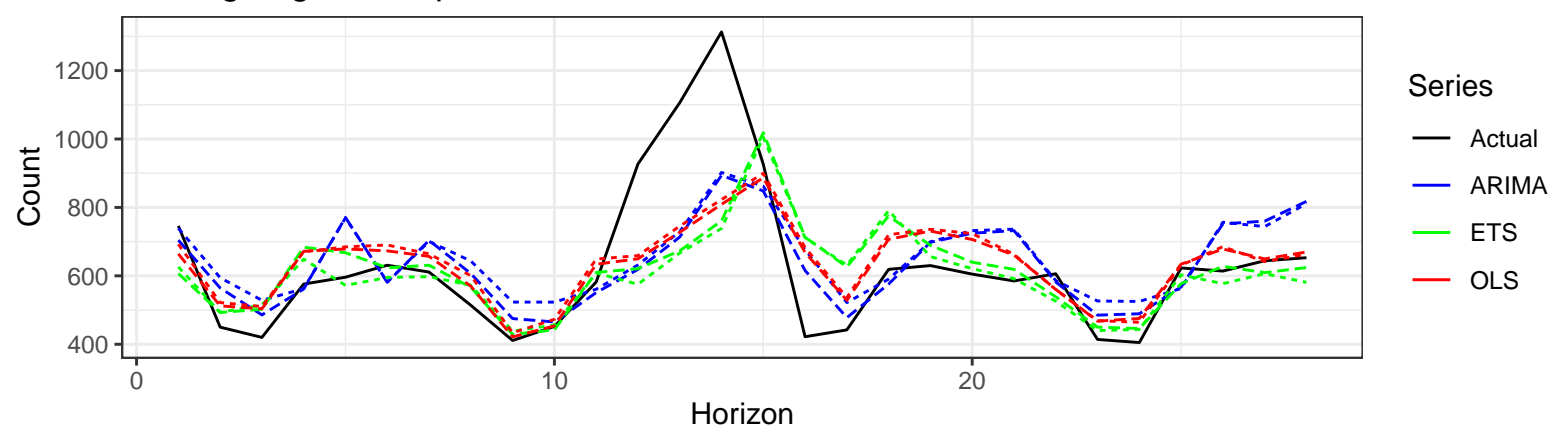

Fixed origin multi-step forecasts

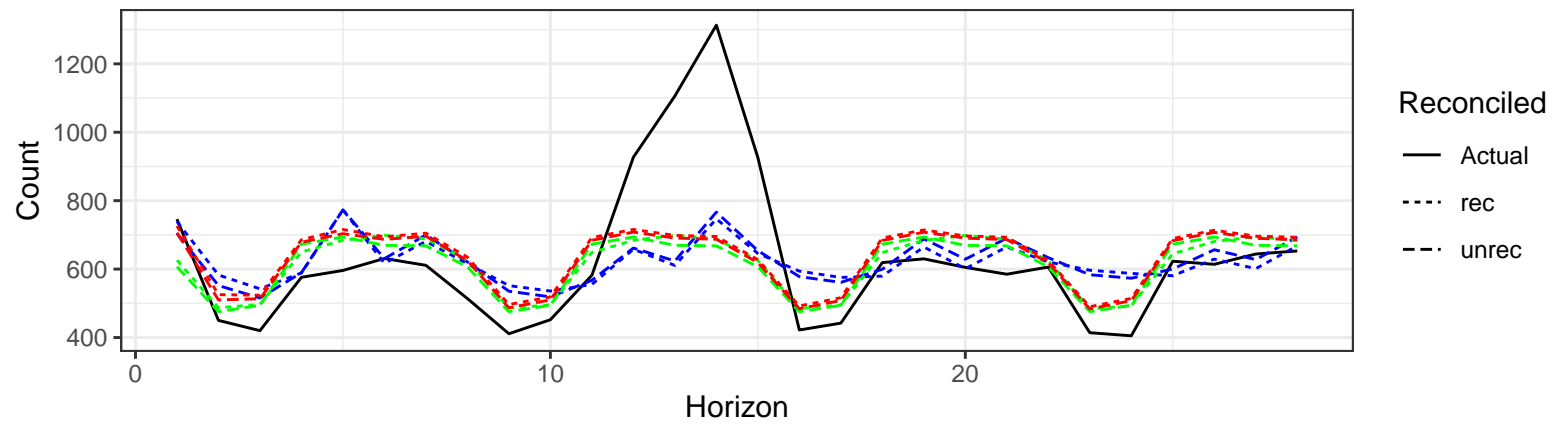

Figure 12: The actual test set for the 'desktopusenPho04' bottom level series compared to the forecasts from reconciled and unreconciled ETS, ARIMA and OLS methods for rolling and fixed origin forecasts of Wikipedia pageviews. 
series, where ETS performs significantly better than ARIMA and OLS. We also note that the reconciliation is less effective. As in the tourism example, in higher levels, series have higher counts and therefore their error magnitudes are larger.

In Figures 11 and 12, we display results for the total and one of the bottom level series, "desktopusenPho04" (desktop-user-english-photo sharing). The plot shows rolling and fixed origin forecast results over the 28 day test set for ETS, ARIMA and OLS, with (solid lines) and without (dashed lines) applying reconciliation. We see that the OLS forecasting model performs close to the other two methods, and reconciliation improves the forecasts.

Table 10 presents the computation times for all three methods. ETS and ARIMA are clearly much more computationally heavy compared with OLS. As in the Australian tourism dataset, running reconciliation does not have much effect on computation time.

Table 10: Computation time (seconds) for ETS, ARIMA and OLS with and without reconciliation Rolling and fixed origin forecasts - Wikipedia dataset

\begin{tabular}{cccccc}
\hline & \multicolumn{4}{c}{ Computation time (secs) } \\
\cline { 2 - 3 } & \multicolumn{2}{c}{ Rolling origin } & & \multicolumn{2}{c}{ Fixed origin } \\
\cline { 2 - 3 } \cline { 5 - 6 } & Unreconciled & Reconciled & & Unreconciled & Reconciled \\
\hline ETS & 27613.08 & 27613.14 & & 971.55 & 971.58 \\
ARIMA & 49419.36 & 49419.39 & & 1769.52 & 1769.56 \\
OLS & 116.27 & 116.31 & & 61.33 & 61.38 \\
\hline
\end{tabular}

\section{Conclusion}

We have proposed a linear model approach to fast forecasting of hierarchical or grouped time series, with accuracy that nearly matches that of forecast methods such as ETS and ARIMA. This is especially useful in large collections of time series, as is typical in hierarchical and grouped structures. Although ETS and ARIMA are advantageous in terms of forecasting power and accuracy, they can be computationally heavy when facing large collections of time series in the hierarchy. An important feature of our model is its ability to easily include external information such as holiday dummies or other external series. We also note that OLS has the additional practical advantage of handling missing data while ETS requires imputation.

Another advantage of our approach is that it can be computed in a single matrix equation (5). This makes it extremely fast and easy to implement, and enables standard results to be derived with minimal effort (e.g., prediction intervals). 
Pennings \& Dalen (2017) proposed another approach for forecasting hierarchical time series using state space models. Although their approach is flexible in handling outliers, missing data and external features, it is less flexible to different kinds of datasets and it is computationally much more complex.

\section{Acknowledgements}

The first and third authors of this research were partially funded by Ministry of Science and Technology (MOST), Taiwan [Grant 106-2420-H-007-019]. 


\section{Appendix A}

We provide boxplots of the scaled forecasted errors for the tourism example. These plots are displayed for both rolling forward and multiple-step-ahead forecasts.

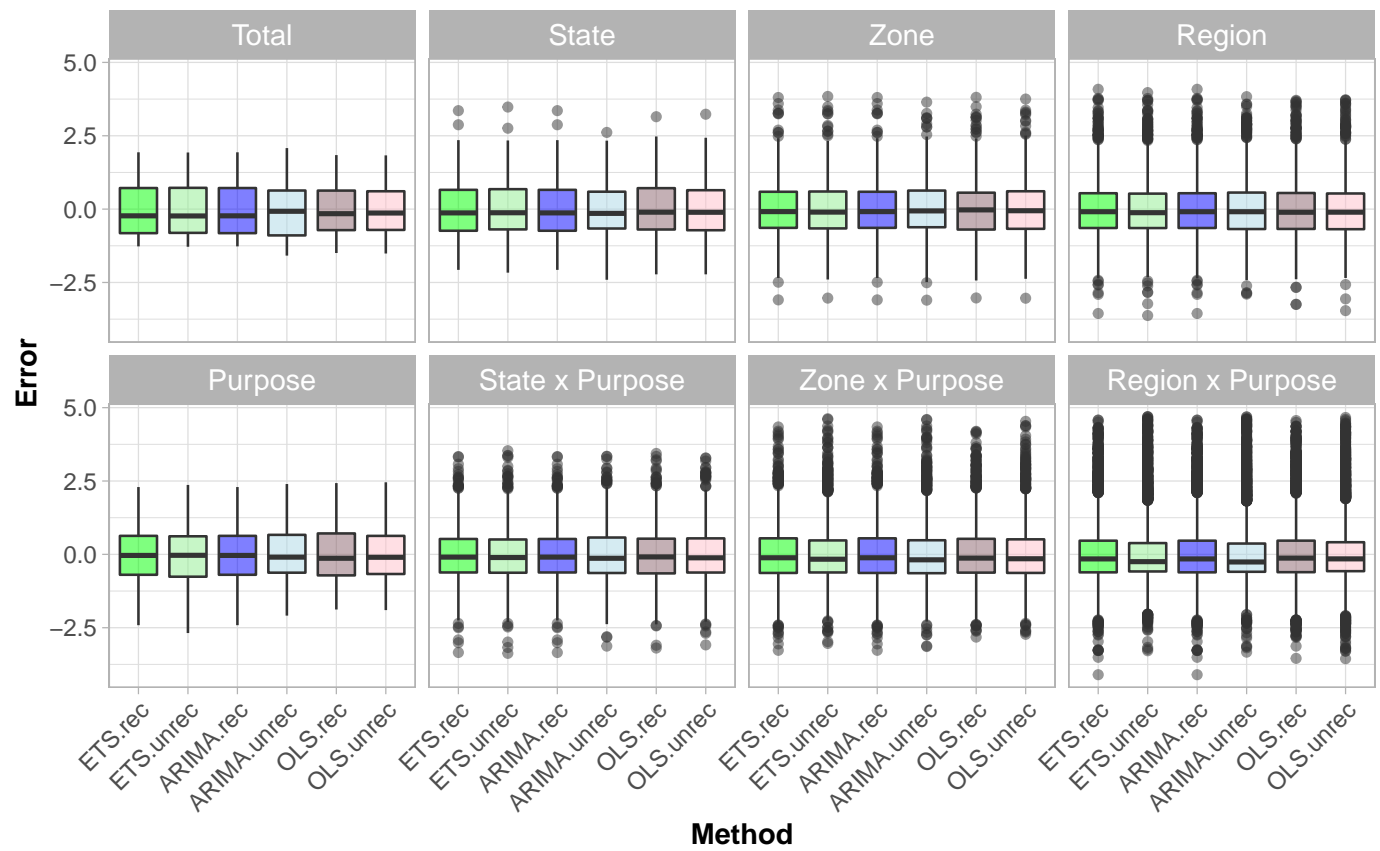

Figure 13: Box plots of scaled forecast errors from reconciled and unreconciled ETS, ARIMA and OLS methods at each hierarchical level for rolling origin 1-step-ahead tourism demand.

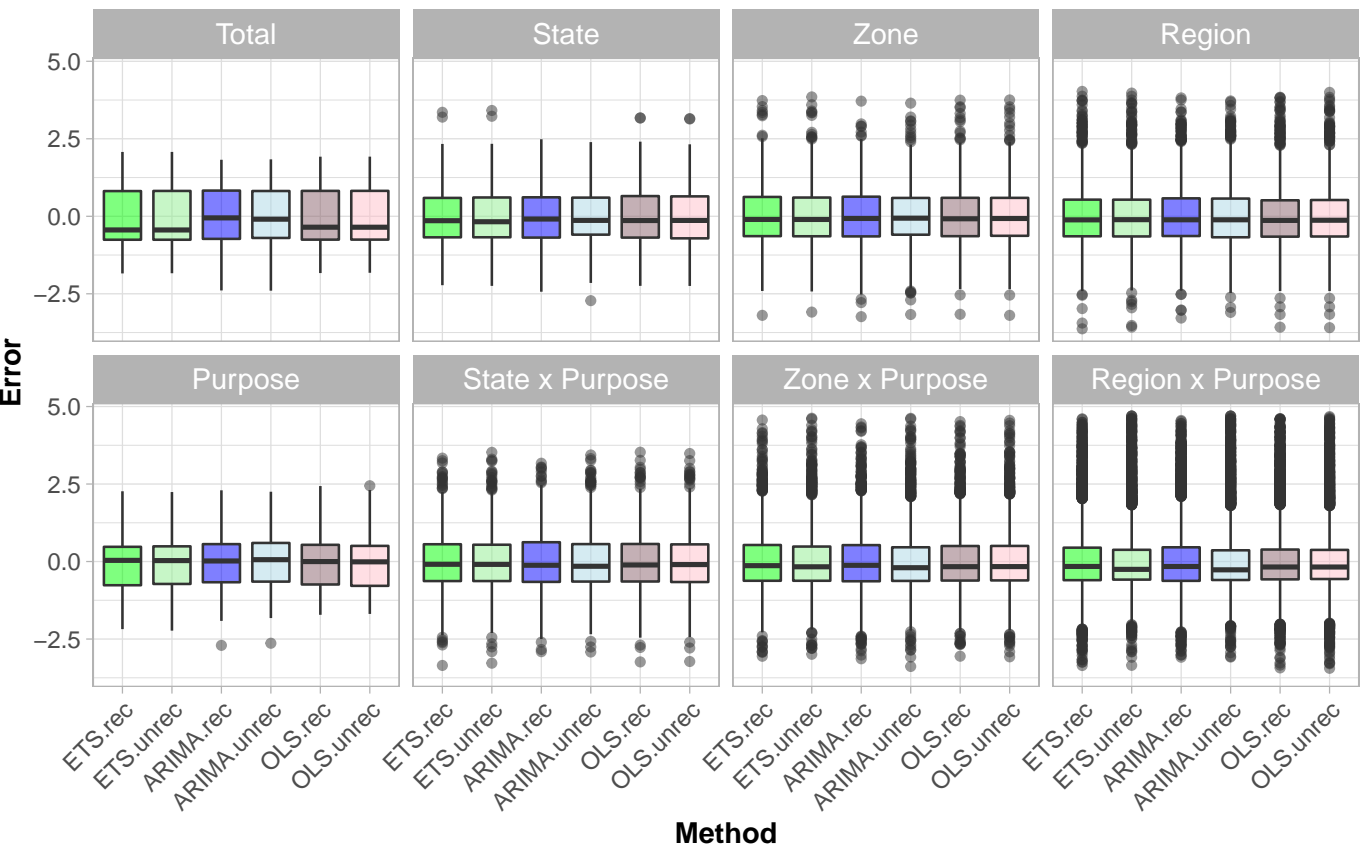

Figure 14: Box plots of scaled forecast errors from reconciled and unreconciled ETS, ARIMA and OLS methods at each hierarchical level for fixed origin multi-step-ahead tourism demand. 


\section{References}

Akaike, H (1998). "Information theory and an extension of the maximum likelihood principle". In: Selected Papers of Hirotugu Akaike. Springer Series in Statistics (Perspectives in Statistics). Springer, pp.199-213.

Ashouri, M, G Shmueli \& CY Sin (2018). Clustering time series by domain-relevant features using model-based trees. Proceedings of the 2018 Data Science, Statistics \& Visualization (DSSV).

Athanasopoulos, G, RA Ahmed \& RJ Hyndman (2009). Hierarchical forecasts for Australian domestic tourism. International Journal of Forecasting 25(1), 146-166.

Fliedner, G (2001). Hierarchical forecasting: issues and use guidelines. Industrial Management $\mathcal{E}$ Data Systems 101(1), 5-12.

Gross, CW \& JE Sohl (1990). Disaggregation methods to expedite product line forecasting. Journal of Forecasting 9(3), 233-254.

Hyndman, RJ, RA Ahmed, G Athanasopoulos \& HL Shang (2011). Optimal combination forecasts for hierarchical time series. Computational Statistics \& Data Analysis 55(9), 2579-2589.

Hyndman, RJ \& G Athanasopoulos (2018). Forecasting: principles and practice. Melbourne, Australia: OTexts. https : //OTexts . org/fpp2.

Kahn, KB (1998). Revisiting top-down versus bottom-up forecasting. The Journal of Business Forecasting 17(2), 14.

Pennings, CL \& J van Dalen (2017). Integrated hierarchical forecasting. European Journal of Operational Research 263(2), 412-418.

Tourism Research Australia (2005). Travel by Australians, September Quarter 2005. Tourism Australia.

Wickramasuriya, SL, G Athanasopoulos \& RJ Hyndman (2019). Optimal forecast reconciliation for hierarchical and grouped time series through trace minimization. Journal of the American Statistical Association 14(526), 804-819. 